\title{
ERAT: A New ERA for Appendicitis Therapy?
}

\author{
Doumit S. BouHaidar ${ }^{1} \cdot$ Muhammad Z. Bawany $^{1} \cdot$ Mitchell L. Schubert $^{1,2}$
}

Published online: 21 September 2016

(c) Springer Science+Business Media New York 2016

Appendicitis, a common cause of acute abdomen, usually occurs in the 2 nd or 3 rd decade of life with an incidence of $233 / 100,000$ [1]. Its pathophysiology is thought to result from appendiceal obstruction due to fecal material, undigested food, foreign body, enlarged lymphoid follicles, stenosis, or twisting of the organ. Initial clinical manifestations include right lower quadrant abdominal pain, nausea, vomiting, and low-grade fever. Classic physical findings such as tenderness at McBurney's point (1.5-2 in. from the anterior superior iliac spine [ASIS] on a straight line from the ASIS to the umbilicus), psoas sign (right lower quadrant pain with passive right hip extension), and Rovsing's sign (pain in the right lower quadrant with palpation of left lower quadrant) either may not be present early in the disease course or are difficult to elicit in an acute abdomen. Leukocytosis is the only consistently abnormal routine laboratory abnormality [2].

Imaging modalities such as computed tomography (CT) (sensitivity, 94-100\%; specificity, 93-100\%) and ultrasonography (sensitivity, 74-100 \%; specificity, 88-99\%) are increasingly used to confirm acute appendicitis and decrease the rate of negative appendectomy [3]. Although preoperative CT scanning reduces the negative appendectomy rate by $\sim 20 \%$, it involves ionizing radiation and may delay surgery. Although diagnostic imaging may be

Doumit S. BouHaidar

doumit.bouhaidar@vcuhealth.org

1 Department of Internal Medicine, Division of Gastroenterology, Virginia Commonwealth University, 1200 E Broad Street, P.O. Box 980341, Richmond, VA 23298-03411, USA

2 Division of Gastroenterology, McGuire VAMC, Richmond, VA, USA unnecessary when the clinical diagnosis is nearly certain, with less diagnostic certainty, the treatment plan is altered in $\sim 60 \%$ of suspected cases.

The 'gold standard' for treatment of acute appendicitis is open or laparoscopic surgical appendectomy. Despite higher cost, laparoscopic appendectomy has gained popularity due to a lower rate of wound infection, less postoperative pain, shorter hospital stays, and lowered morbidity $[4,5]$. A non-operative approach to acute appendicitis has also been suggested: In a randomized controlled trial comparing antibiotics alone versus appendectomy $(\mathrm{n}=243)$, the complication rate was lower with antibiotics (4.9 vs. $8.4 \%$ ) but the recurrence rate was $26 \%$ within a year [6].

Endoscopic retrograde appendicitis therapy (ERAT) is a new and minimally invasive technique for the diagnosis and treatment of acute appendicitis patterned after the success that endoscopic retrograde cholangiopancreatography (ERCP) achieved in the treatment of acute cholangitis. For ERAT, a colonoscope is used to access the appendiceal orifice and inspect the area for signs of acute appendicitis such as hyperemia, edema, pus, or impacted appendicolith. A transparent cap attached to the tip of the colonoscope improves visualization of the appendiceal orifice by flattening folds when pressed against the mucosa. The appendiceal lumen is cannulated with a 0.035 -in. in diameter ERCP guidewire to facilitate dye injection for fluoroscopic imaging and to obtain access for therapy. Definitive treatment includes irrigation, appendicolith removal, and plastic stent placement for appendiceal orifice stenosis. The bowel can be prepared for the procedure with $500 \mathrm{ml}$ of a normal saline solution delivered as a lowpressure enema.

The initial report of appendicitis diagnosed and treated with ERAT was in 1995 [7]. In 2012, a small pilot study 
from China was published [8]; the same Chinese group published, in 2015, a multicenter retrospective study of 41 patients with suspected acute uncomplicated appendicitis managed with ERAT. Of 34 patients $(83 \%)$ diagnosed with acute appendicitis, $97 \%$ were successfully treated with ERAT [9].

In this issue of Digestive Diseases and Sciences, Li et al. [10] report retrospectively on 21 patients who underwent ERAT to diagnose and treat acute appendicitis. The patients underwent colonoscopic direct visualization of the appendiceal orifice as well as endoscopic retrograde appendicography (ERA), the latter involving injection of a soluble contrast agent with fluoroscopic guidance into the appendix to evaluate for radiographic features of appendicitis such as irregular contour, enlarged appendiceal lumen ( $\geq 5 \mathrm{~mm}$ ), appendicolith, and luminal stenosis.

The authors are to be commended for reporting a relatively large case series illustrating the utility of ERAT to diagnose and treat acute appendicitis. The sample size is sufficient to serve as a basis for the design of a prospective randomized study. The diagnosis of acute appendicitis was confirmed in 20 of $21(95 \%)$ patients-15 (75\%) by endoscopic inspection and $5(25 \%)$ by ERA. The reason the five patients diagnosed by ERA had no endoscopic abnormalities is unknown, but it is possible that inflammation was limited to the tip or the body of the appendix. Fluoroscopy also enabled therapy to be tailored to the findings, for instance placing a plastic stent for appendiceal orifice stenosis.

Although the cannulation rate was $100 \%$ and there were no perforations, such excellent results may not be replicable in less experienced hands. Appendiceal perforation is a potential complication that may occur as a result of a difficult cannulation or excess insufflation of a diseased appendix. Mean operative time for ERAT was $50 \mathrm{~min}$, longer than that required for laparoscopic appendectomy (mean, 10 min; CI 6-15 min) [11]. Nevertheless, ERAT not only avoided surgery, but also provided immediate pain relief and, compared to surgery, a shorter course of antibiotic therapy, and a quicker recovery.

Radiographic imaging is required prior to performing ERAT. Patients were excluded from ERAT if the initial radiologic imaging revealed a perforated appendix, a periappendiceal abscess, or if the patient was allergic to the contrast agent. The quality of the bowel preparation using low-pressure saline enemas was not mentioned in the study, but appeared to be sufficient to perform the procedure.

Additional advantages of ERAT include inspection of the entire colon and preservation of the appendix. Significant pathologic findings such as polyps can be subsequently or concurrently managed. In the current study, a large polyp was removed by endoscopic mucosal resection. Since the appendix may serve a beneficial immunologic function and support the gut microbiome by helping recolonize the colon with commensal flora, its preservation appears to be desirable [12].

How should a patient with suspected acute appendicitis be managed currently? Initially, routine measures such as obtaining a careful history and performing a physical examination, and obtaining routine laboratory tests and radiologic imaging (CT and/or ultrasound) are required. In light of the initial data obtained, alternative diagnoses should be considered such as acute diverticulitis, Crohn's disease, pelvic inflammatory disease, ectopic pregnancy, ureteral stone, and endometriosis. Although surgery remains the 'gold standard' against which all other interventions should be compared, available studies suggest that ERAT, in those experienced with the procedure, is a safe and effective diagnostic and therapeutic procedure with a reported complication rate of $\sim 5 \%$ with no need for emergent surgical appendectomy in cases amenable to endoscopic treatment. Successful completion of a prospective, randomized, controlled trial comparing surgery versus ERAT is needed in order to determine whether ERAT will supplant existing interventions in the treatment of acute appendicitis.

\section{References}

1. Addiss DG, Shaffer N, Fowler BS, Tauxe RV. The epidemiology of appendicitis and appendectomy in the United States. Am J Epidemiol. 1990;132:910-925.

2. Gronroos JM, Gronroos P. Leucocyte count and C-reactive protein in the diagnosis of acute appendicitis. $B r \quad J$ Surg. 1999;86:501-504.

3. Wagner PL, Eachempati SR, Soe K, Pieracci FM, Shou J, Barie PS. Defining the current negative appendectomy rate: for whom is preoperative computed tomography making an impact? Surgery. 2008;144:276-282.

4. Harrell AG, Lincourt AE, Novitsky YW, et al. Advantages of laparoscopic appendectomy in the elderly. Am Surg. 2006;72:474-480.

5. Sporn E, Petroski GF, Mancini GJ, Astudillo JA, Miedema BW, Thaler K. Laparoscopic appendectomy-is it worth the cost? Trend analysis in the US from 2000 to 2005. J Am Coll Surg. 2009;208:179-185.

6. Vons C, Barry C, Maitre S, et al. Amoxicillin plus clavulanic acid versus appendicectomy for treatment of acute uncomplicated appendicitis: an open-label, non-inferiority, randomised controlled trial. Lancet. 2011;377:1573-1579.

7. Said M, Ledochowski M, Dietze O, Simader H. Colonoscopic diagnosis and treatment of acute appendicitis. Eur J Gastroenterol Hepatol. 1995;7:569-571.

8. Liu BR, Song JT, Han FY, Li H, et al. Endoscopic retrograde appendicitis therapy: a pilot minimally invasive technique (with videos). Gastrointest Endosc. 2012;76:243-247.

9. Liu BR, Ma X, Feng J, et al. Endoscopic retrograde appendicitis therapy (ERAT): a multicenter retrospective study in China. Surg Endosc. 2015;29:905-909. 
10. Li Y, Mi C, Li W, She J. Diagnosis of acute appendicitis by endoscopic retrograde appendicitis therapy (ERAT): combination of colonoscopy and endoscopic retrograde appendicography. Dig Dis Sci. (Epub ahead of print). doi:10.1007/s10620-016-4245-8.

11. Sauerland S, Jaschinski T, Neugebauer EA. Laparoscopic versus open surgery for suspected appendicitis. Cochrane Database Syst Rev. 2010. doi:10.1002/14651858
12. Kooij IA, Sahami S, Meijer SL, Buskens CJ, Te Velde AA. The immunology of the vermiform appendix: a review of the literature. Clin Exp Immunol. 2016;186:1-9. doi:10.1111/cei. 12821 